\title{
Electron Beam Control of Dopants in 2D and 3D Materials
}

Andrew R. Lupini ${ }^{1}$, Bethany Hudak ${ }^{2}$, Stephen Jesse ${ }^{3}$, Jiaming Song ${ }^{4}$, Ondrej Dyck ${ }^{1}$, Paul Snijders ${ }^{5}$ and Sergei Kalinin 6

${ }^{1}$ Oak Ridge National Laboratory, United States, ${ }^{2}$ U.S. Naval Research Laboratory, District of Columbia, United States, ${ }^{3}$ Center for Nanophase Materials Sciences, Oak Ridge National Laboratory, United States, ${ }^{4}$ School of Physics, Northwest University, Xi'an, China, United States, ${ }^{5}$ Materials Science and Technology Division, Oak Ridge National Laboratory, United States, ${ }^{6}$ Center for Nanophase Materials Sciences, Oak Ridge National Laboratory, Oak Ridge, Tennessee, United States

The properties of many modern materials are dominated by the type, number, and location of dopants. This situation is exemplified by proposals for new quantum materials and devices, where their entire functionality might depend on the precise placement of single dopant atoms. The emerging challenges are therefore how to control and determine the dopant locations and how they affect the physical properties.

High-resolution high-angle annular dark field (HAADF) scanning transmission electron microscopy (STEM) provides a powerful method to address both of these challenges at the single atomic scale. This imaging mode is able to locate single atoms in both two-dimensional (2D) materials, such as graphene, as well as 3D single crystals, although the technical challenges are different in both cases. In monolayer materials, every atom can potentially be identified [1] (as shown in Fig. 1). However, one problem is that these materials are easily damaged at the accelerating voltages used in conventional electron microscopy, meaning that the voltage has to be chosen to reduce the amount of damage, and switching the voltage typically causes long-term drift. Advances in both hardware and software from microscope manufacturers and methods such as maintaining constant power in the lenses, even when changing accelerating voltage [2] help to address these problems. Such advances are enabling a move from a regime where the microscope is operated in a standard mode to one in which the scientist is free to select the beam energy as appropriate for the experiment. This ability raises several new experimental possibilities, such as using the atomic-sized probe in a STEM to control single atoms inside 2D materials [3,4], much as scanning tunneling microscopy has been able to do for atoms on surfaces.

For 3D materials, such as single crystals, the HAADF mode is best suited for finding dopants in cases where there is a large atomic number difference between a light matrix and a heavy dopant. The complication for finding a single dopant is that in almost every sample, the surface is not atomically smooth or clean. Variations in sample thickness, and even subtle changes in surface structure closely resemble the contrast changes that might be expected for light dopants. Thus locating dopants with a small atomic number difference or accurately determining the number of light atoms in a column remain as ongoing challenges, especially when single atoms can diffuse rapidly [5]. Similar to the case for 2D materials, the electron beam transfers energy to the sample, which can cause even dopants inside the sample to move (as shown in Fig. 2). This movement has recently been exploited to allow two-dimensional control of dopants in 3D materials [6]. Although several of the important factors were identified, the mechanism for the dopant control was not fully elucidated, leaving several important questions unanswered, such as which other dopants might be manipulated in the same way. Through-focus-series methods offer a way to pinpoint [7] single atoms in all 3-dimensions and close control of focusing and exploitation of electron channeling could potentially provide control of dopants in the depth direction. 
Another route to detect local changes in the sample might be to rely on other imaging modes. Single dopants can potentially be found using EELS or X-ray detection, but not every atom provides a large cross-section. A new generation of fast and sensitive detectors allows 4D image acquisition [8] and more efficient use of almost all the electrons that have interacted with the sample $[9,10]$. Similarly, it might be possible to map the functionality, such as conductivity at high spatial resolution. Recent results have shown that secondary-electron electron-beam-induced current imaging can detect even single monolayers of graphene [11]. Perhaps the next challenge will be not just to use the electron beam to image dopants, but to combine with machine learning and functional imaging modes to unveil and control their physical properties atom-by-atom [12,13].

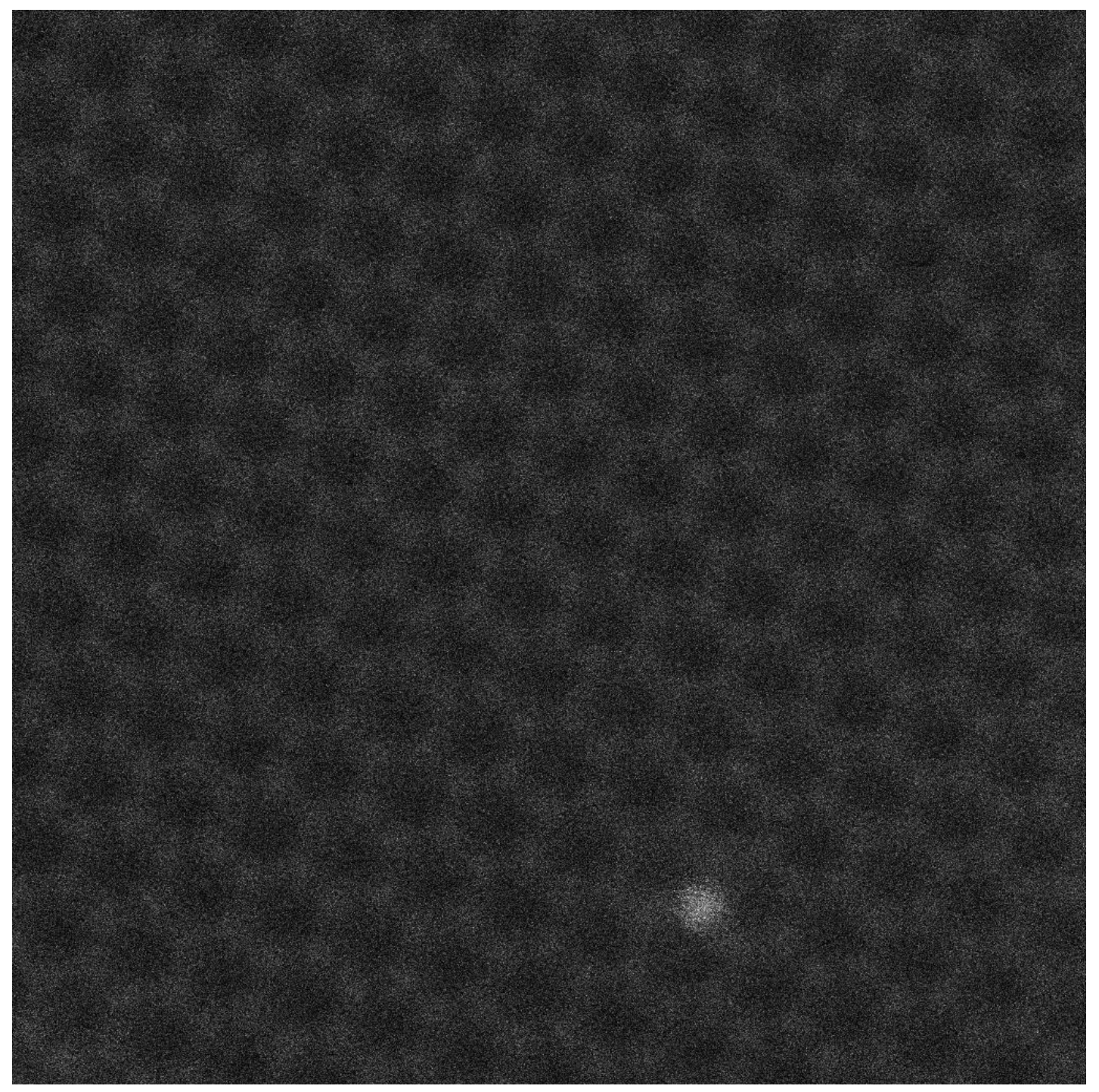

Figure 1. Single Si atom in monolayer graphene imaged at $60 \mathrm{kV}$ to reduce damage and dopant movement. 

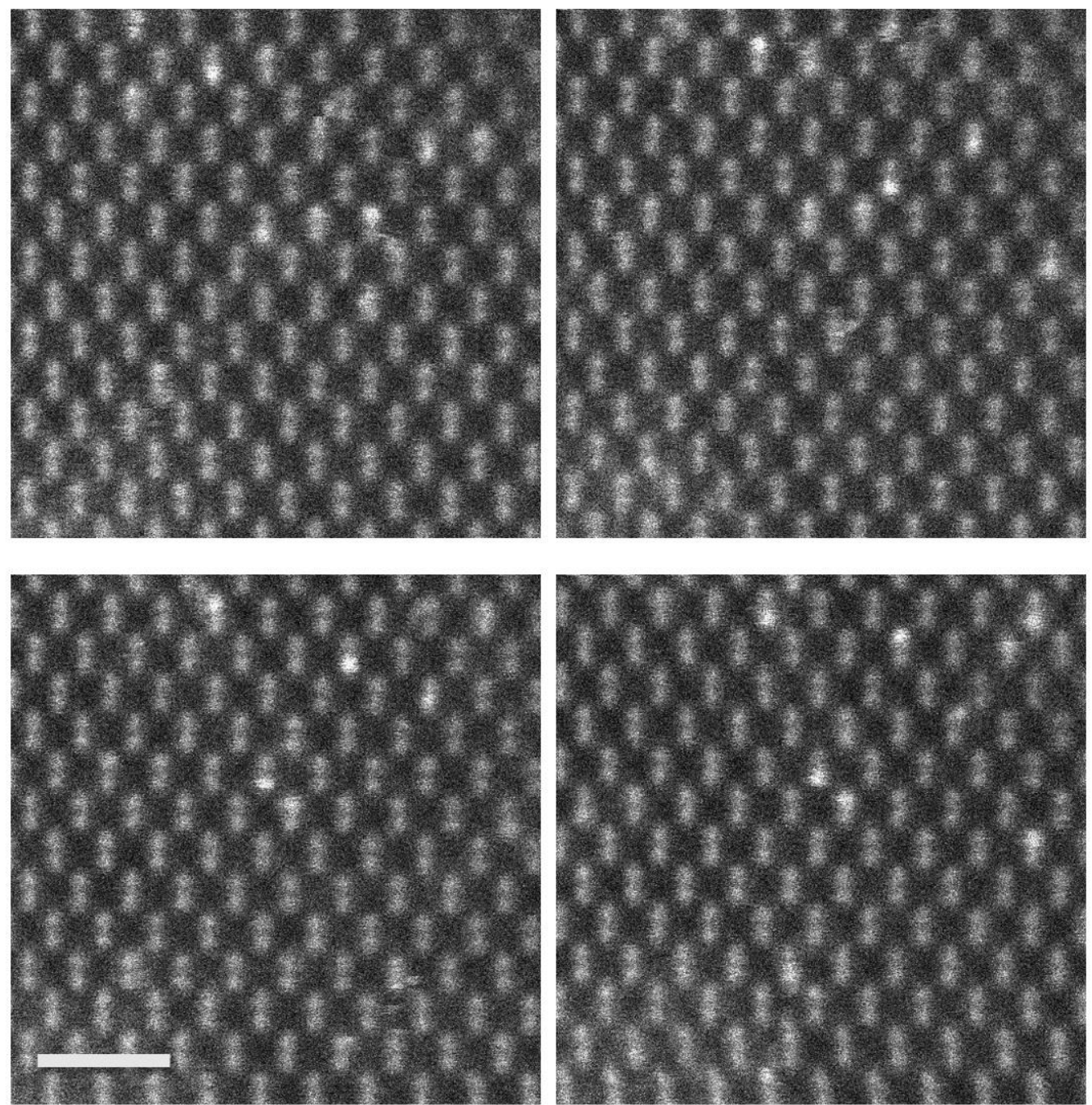

Figure 2. High-angle annular dark field (HAADF) images showing single heavy Bi dopant atoms in $\mathrm{Si}[110]$. A $200 \mathrm{kV}$ electron beam was used to record an image sequence, causing dopants atoms to move from column to column in an uncontrolled manner and to occasionally occupy interstitial locations. Scale bar represents $1 \mathrm{~nm}$.

\section{References}

[1] O.L Krivanek et al, Nature 464 (2010) 571-574

[2] O. Dyck et al., Ultramicroscopy 211 (2020) 112949

[3] O. Dyck et al., Applied Physics Letters 111 (2017) 113104

[4] T Susi et al., Ultramicroscopy 180 (2017) 163

[5] R. Ishikawa et al., Phys Rev Lett 113 (15) (2014) 15550

[6] B.M. Hudak et al., ACS Nano 12 (2018) 5873

[7] R. Ishikawa et al., Phys Rev Applied 13 (2020) 034064

[8] C. Ophus, Microscopy and Microanalysis 25 (2019) 563-582

[9] T.J. Pennycook et al., Ultramicroscopy 151 (2015) 160-167

[10] A.R. Lupini, M. Chi, S. Jesse, Journal of Microscopy 263 (2016) 43-50

[11] O. Dyck et al, Small Methods, (2021) 2000950 
[12] M.P. Oxley et al., Mach. Learn.: Sci. Technol. 1 (2020) 04LT01

[13] Work supported by the U.S. Department of Energy, Office of Science, Basic Energy Sciences, Division of Materials Science and Engineering, and sample growth by the Laboratory Directed Research and Development Program of Oak Ridge National Laboratory, managed by UT-Battelle, LLC for the U.S. Department of Energy, and was performed at the Oak Ridge National Laboratory's Center for Nanophase Materials Sciences (CNMS), a U.S. Department of Energy Office of Science User Facility. 